\title{
HIV-1 Capsid Function is Regulated by Dynamics: Quantitative Atomic-Resolution Insights by Integrating Magic-Angle-Spinning NMR, QM/MM, and MD
}

Huilan Zhang ${ }^{1,2, \#}$, Guangjin Hou ${ }^{1,2, \#}$, Manman Lu, ${ }^{1,2}$, Jinwoo Ahn²,3, In-Ja L. Byeon ${ }^{2,3}$, Christopher J. Langmead ${ }^{4}$, Juan R. Perilla ${ }^{5}$, Ivan Hung ${ }^{6}$, Peter L. Gor'kov ${ }^{6}$, Zhehong Gan ${ }^{6}$, William W. Brey ${ }^{6}$, David A. Case ${ }^{7}$, Klaus Schulten ${ }^{5}$, Angela M. Gronenborn ${ }^{2,3^{*}}$, and Tatyana Polenova ${ }^{1,2^{*}}$

\footnotetext{
${ }^{1}$ Department of Chemistry and Biochemistry, University of Delaware, Newark, Delaware 19716, United States; ${ }^{2}$ Pittsburgh Center for HIV Protein Interactions, University of Pittsburgh School of Medicine, 1051 Biomedical Science Tower 3, 3501 Fifth Ave., Pittsburgh, PA 15261, United States; ${ }^{3}$ Department of Structural Biology, University of Pittsburgh School of Medicine, 3501 Fifth Ave., Pittsburgh, PA 15261, United States; ${ }^{4}$ Computer Science Department, Carnegie Mellon University, Gates Hillman Center, 5000 Forbes Avenue, Pittsburgh, PA, United States; ;

${ }^{5}$ Department of Physics and Beckman Institute for Advanced Science and Technology University of Illinois at UrbanaChampaign, Urbana, Illinois 61801; ${ }^{6}$ National High Magnetic Field Laboratory, Florida State University, Tallahassee, FL, 32310, United States; ${ }^{7}$ Department of Chemistry and Chemical Biology, Rutgers University, 174 Frelinghuysen Road, Piscataway, NJ 08854-8087, United States
}

\#These authors have contributed equally

${ }^{*}$ Corresponding authors: Tatyana Polenova, Department of Chemistry and Biochemistry, University of Delaware, Newark, DE, USA, Tel.: (302) 831-1968; Email: tpolenov@udel.edu; Angela M. Gronenborn, Department of Structural Biology, University of Pittsburgh School of Medicine, 3501 Fifth Ave., Pittsburgh, PA 15260, USA, Tel.: (412) 648-9959; Email: amg100@pitt.edu

Classification: Biological Sciences- Biophysics and Computational Biology

Keywords: magic-angle spinning NMR, HIV-1 capsid, CA protein assemblies, HIV-AIDS, conformational dynamics, chemical shift anisotropy, quantum mechanics/molecular mechanics 


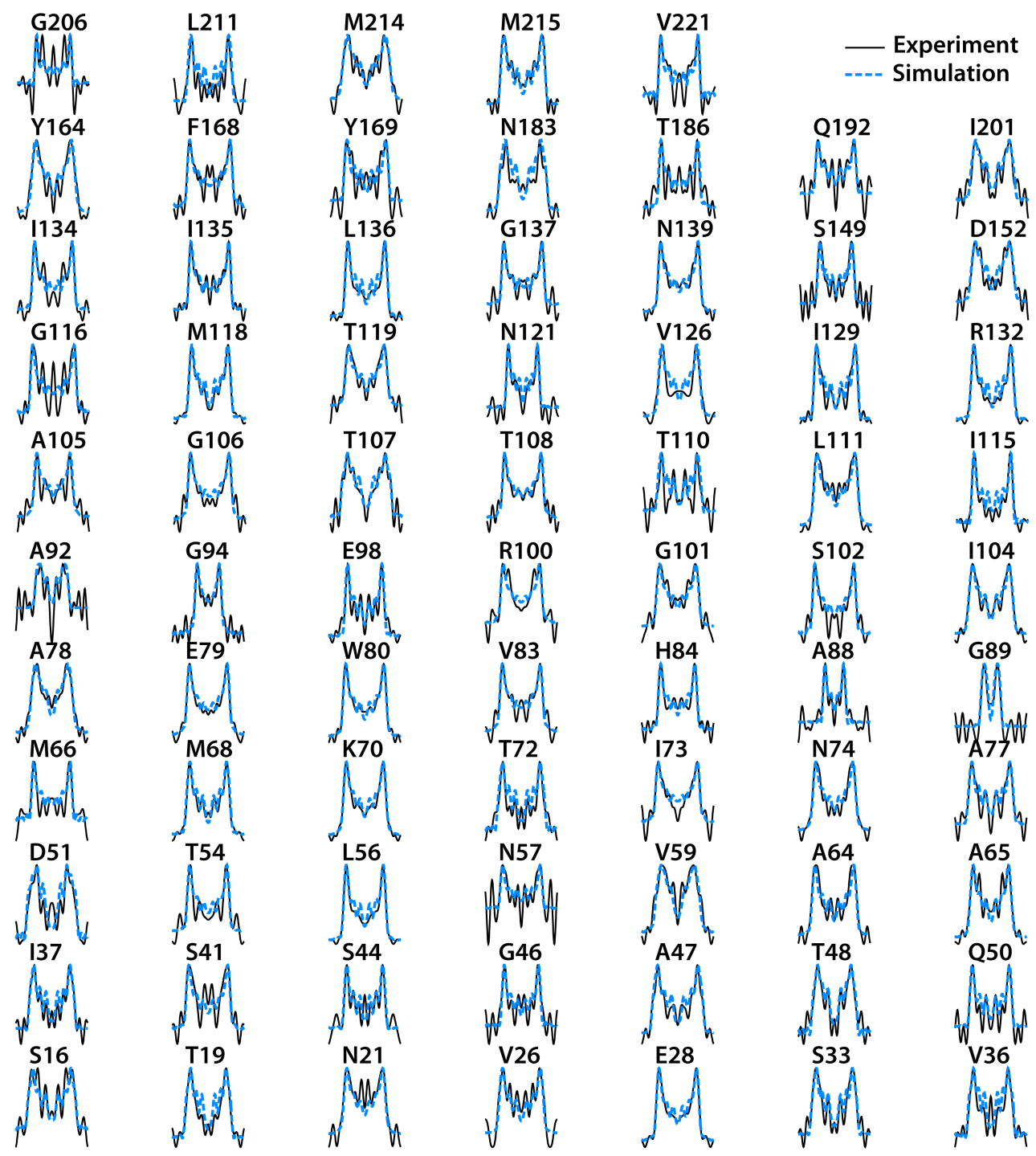

Figure S1. Experimental (solid black lines) and simulated (dashed blue lines) ${ }^{15} \mathrm{~N}$ CSA lineshapes for different residues in tubular assemblies of CA HXB2 extracted from the R8 ${ }_{1}{ }^{3}$ RNCSA 3D spectra, recorded at the magnetic field of $21.1 \mathrm{~T}$ and the MAS frequency of $14 \mathrm{kHz}$. 


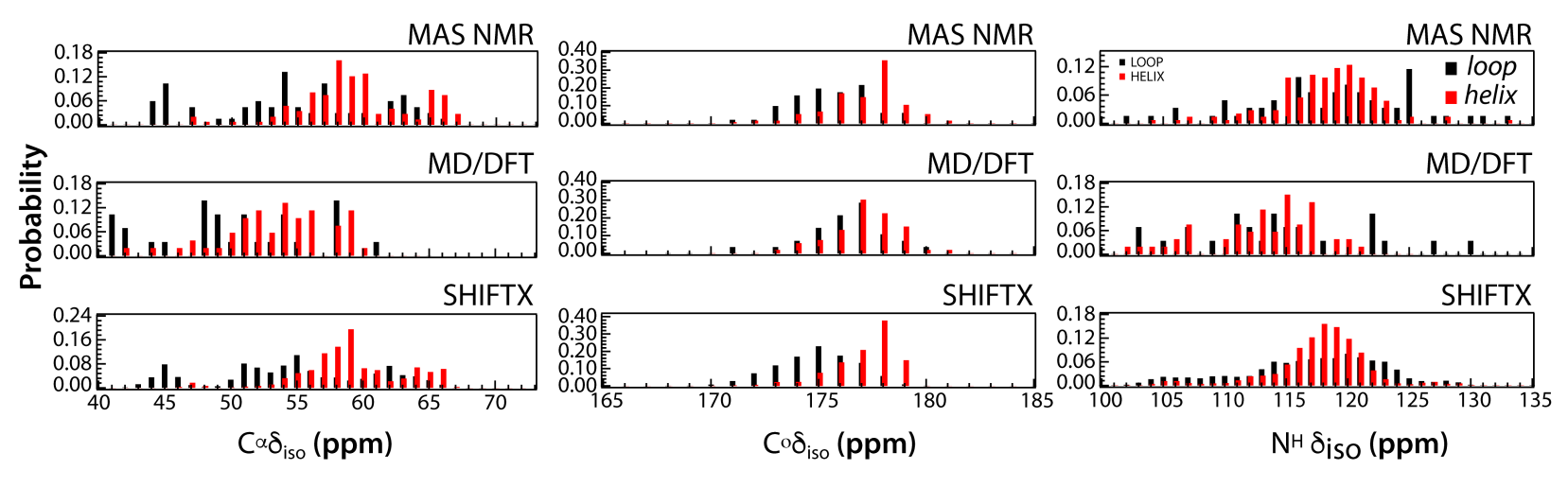

Figure S2. Distribution plots for the isotropic ${ }^{13} \mathrm{C}^{\alpha}$ (left), ${ }^{13} \mathrm{C}^{\circ}$ (middle), and ${ }^{15} \mathrm{~N}^{\mathrm{H}}$ (right) chemical shifts in HIV-1 CA assemblies. Top: experimental MAS NMR; middle, calculated from MD/DFT; bottom, calculated by SHIFTX as the averaged values over the MD trajectory. The distributions for helical regions are shown in red, for loops- in black. 

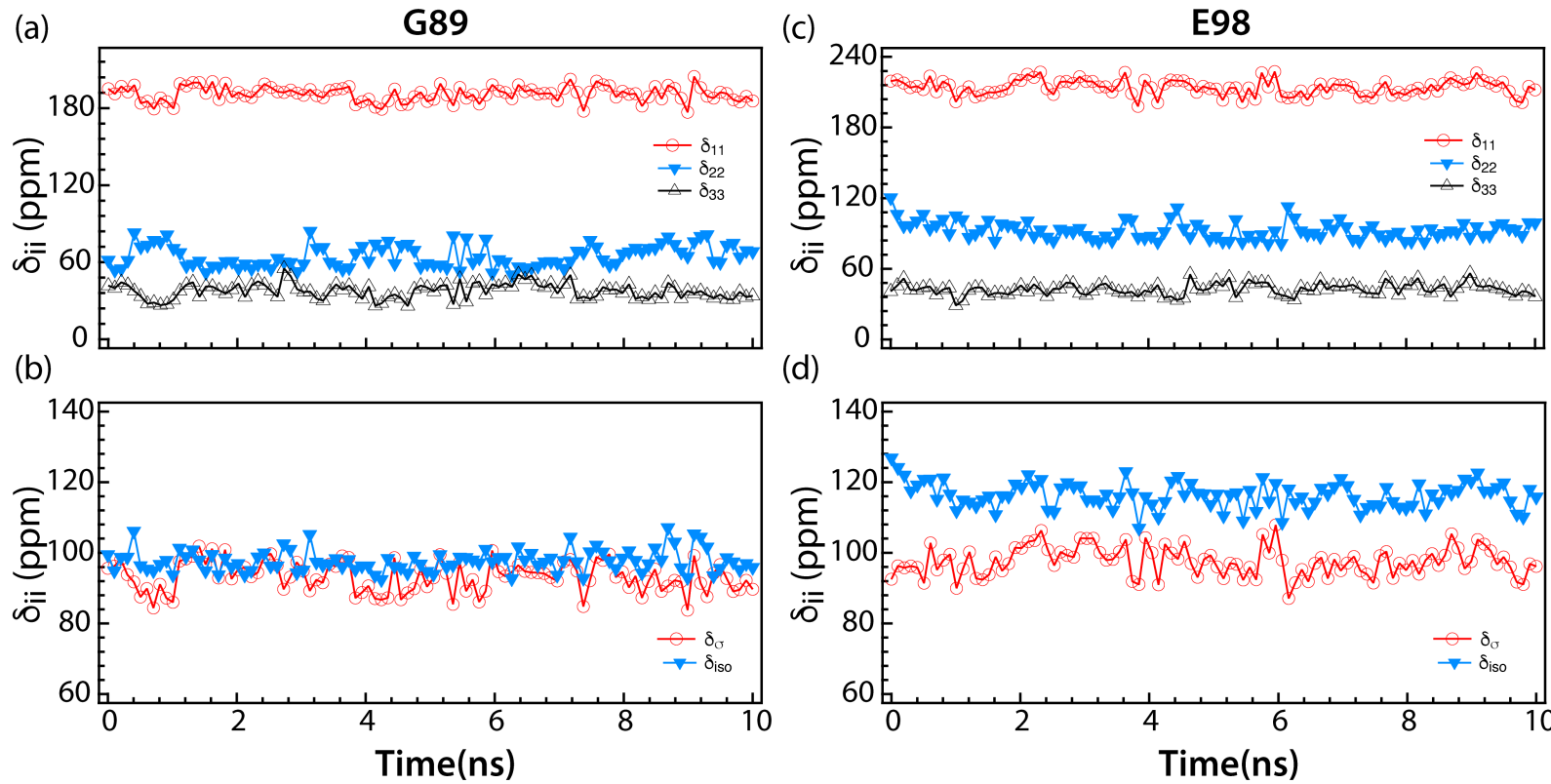

(d)

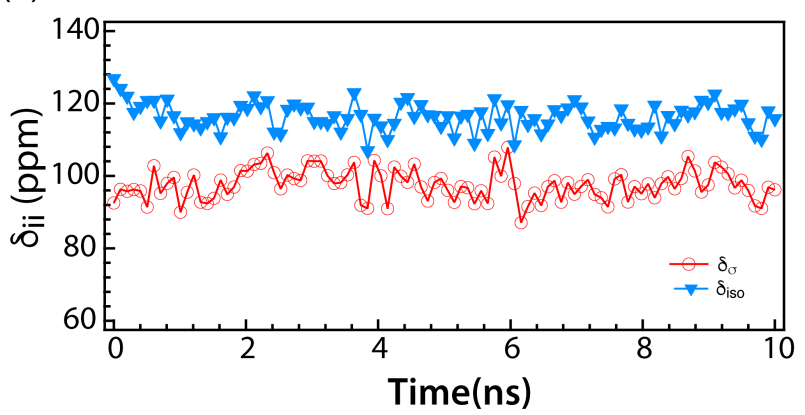

Figure S3. Principal components of ${ }^{15} \mathrm{~N}$ CSA tensor, $\delta_{i i}, \delta_{\sigma}$, and $\delta_{\text {iso, }}$, calculated along the MD trajectory, for selected CA residues: $G 89(a, c)$ and E98 (b, d). For the calculations, 100 frames were used from the first 10 ns of the 100 -ns MD trajectory. 

(a) $\delta_{\sigma}=100 \mathrm{ppm}, \eta=1.0$
(b) $\delta_{\sigma}=100 \mathrm{ppm}, \eta=0.5$

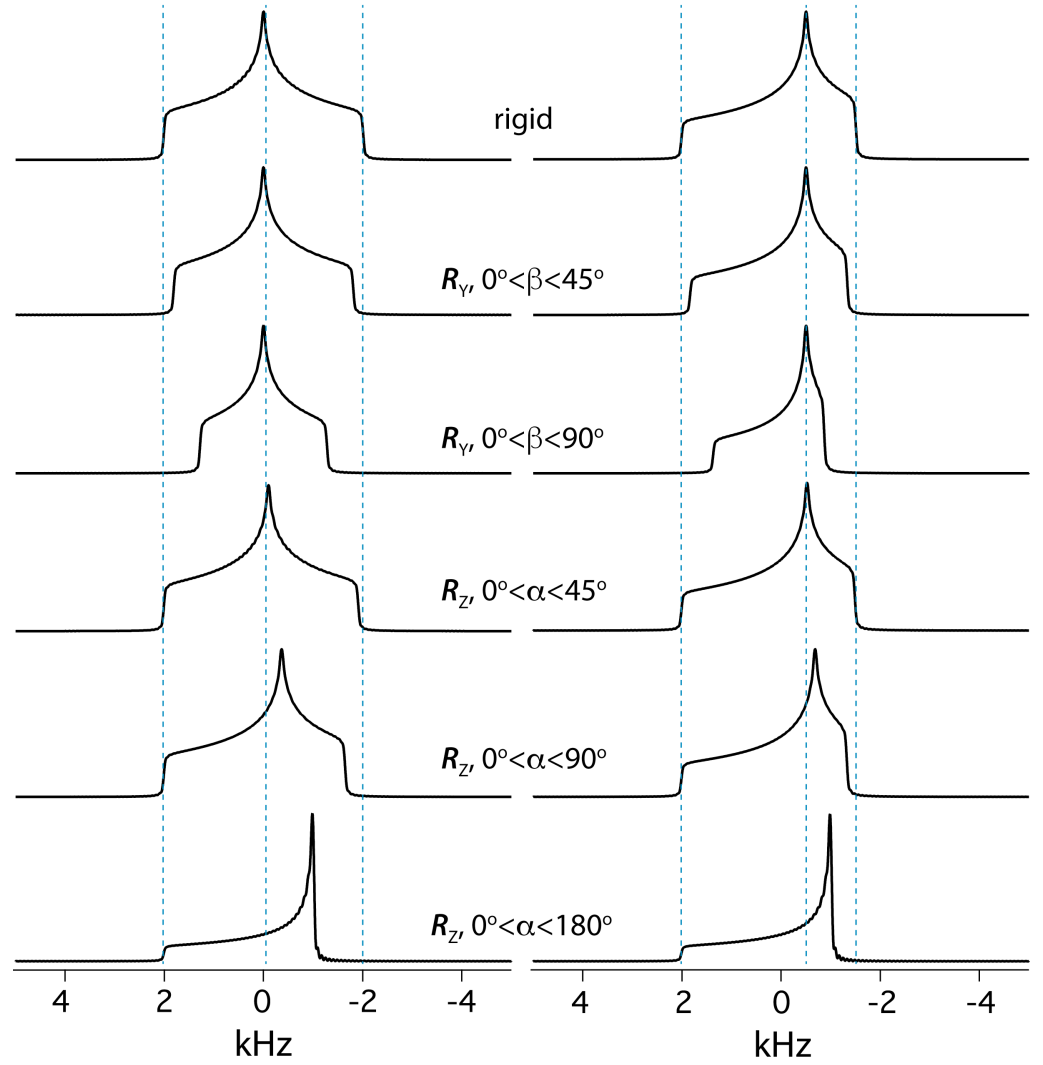

Figure S4. Simulated rigid and motionally reduced ${ }^{15} \mathrm{~N}$ CSA line shapes for the sites with the following CSA NMR parameters: (a) $\delta_{\sigma}=100 \mathrm{ppm}$ and $\eta=1.0$; (b) $\delta_{\sigma}=100 \mathrm{ppm}$ and $\eta=0.5$. The Euler angles are indicated next to each line shape. 

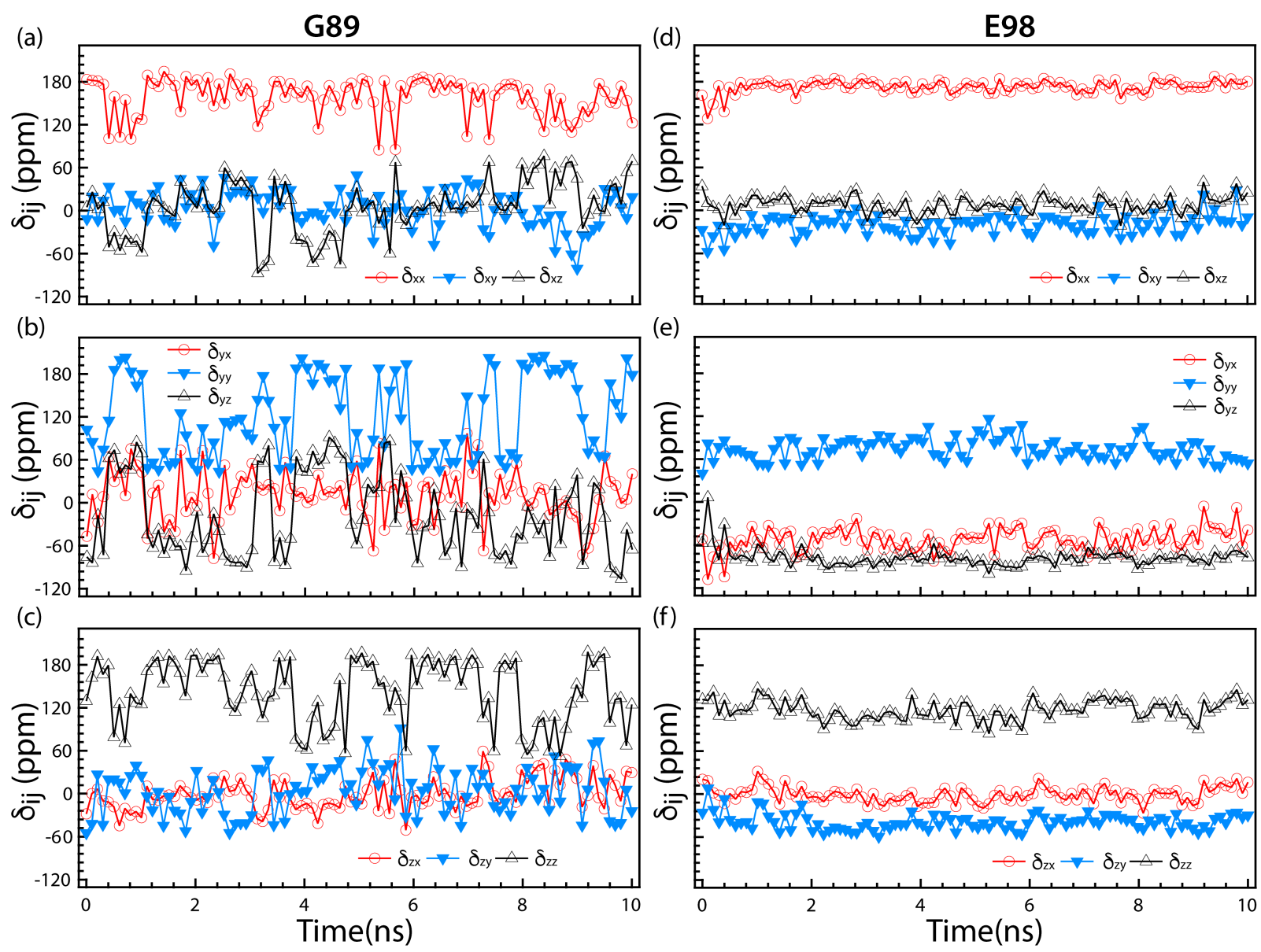

Figure S5. Individual components of ${ }^{15} \mathrm{~N}$ CSA tensor $\delta_{i j}$ (molecular fixed frame representation), calculated along the MD trajectory, for selected CA residues: G89 (a-c) and E98 (d-f). For the calculations, 100 frames were used from the first 10 ns of the 100-ns MD trajectory. 


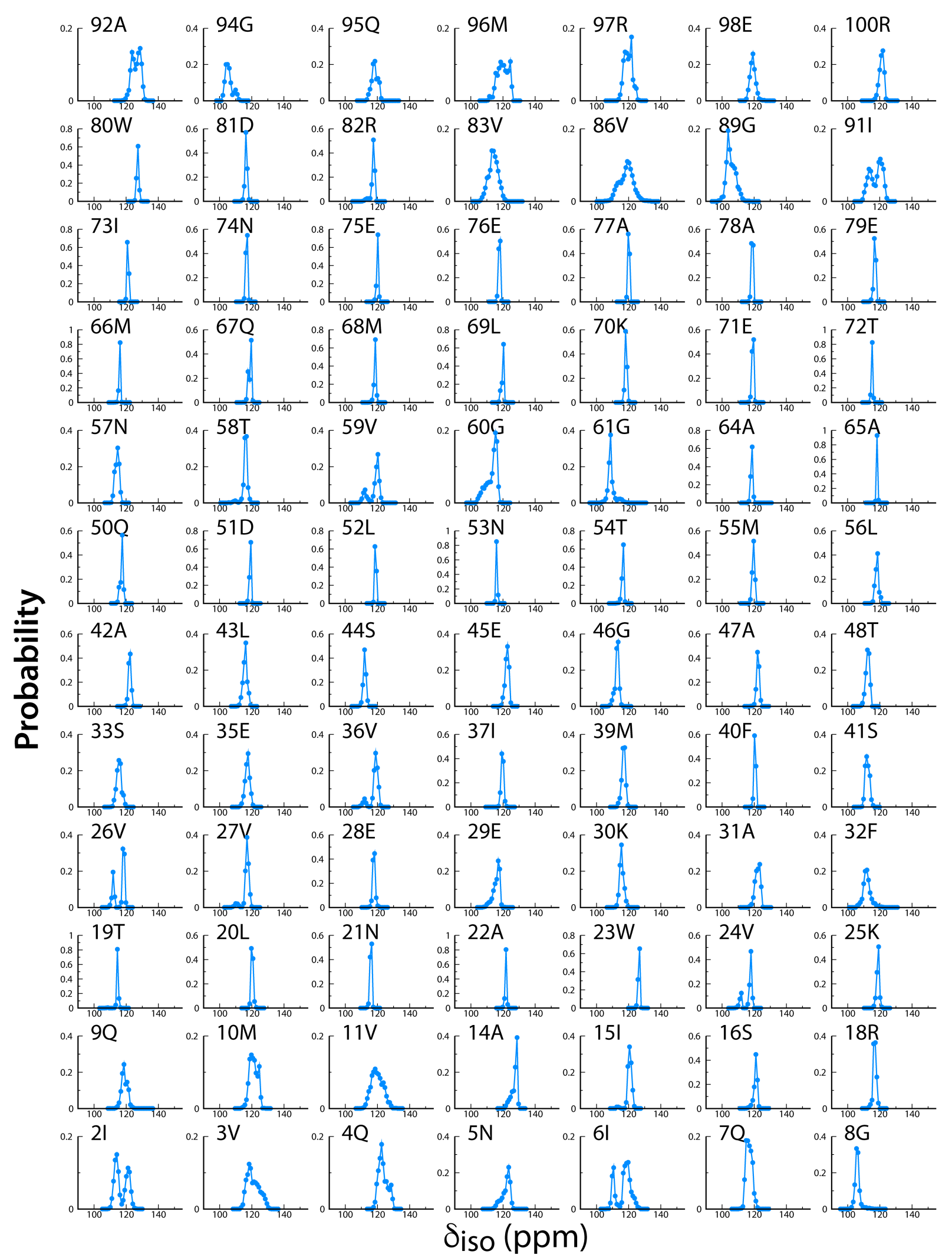

Figure S6. Probability distributions of ${ }^{15} \mathrm{~N}$ isotropic chemical shifts of HIV-1 CA calculated by Shiftx based on 5000 frames extracted from 100 ns MD simulation. 


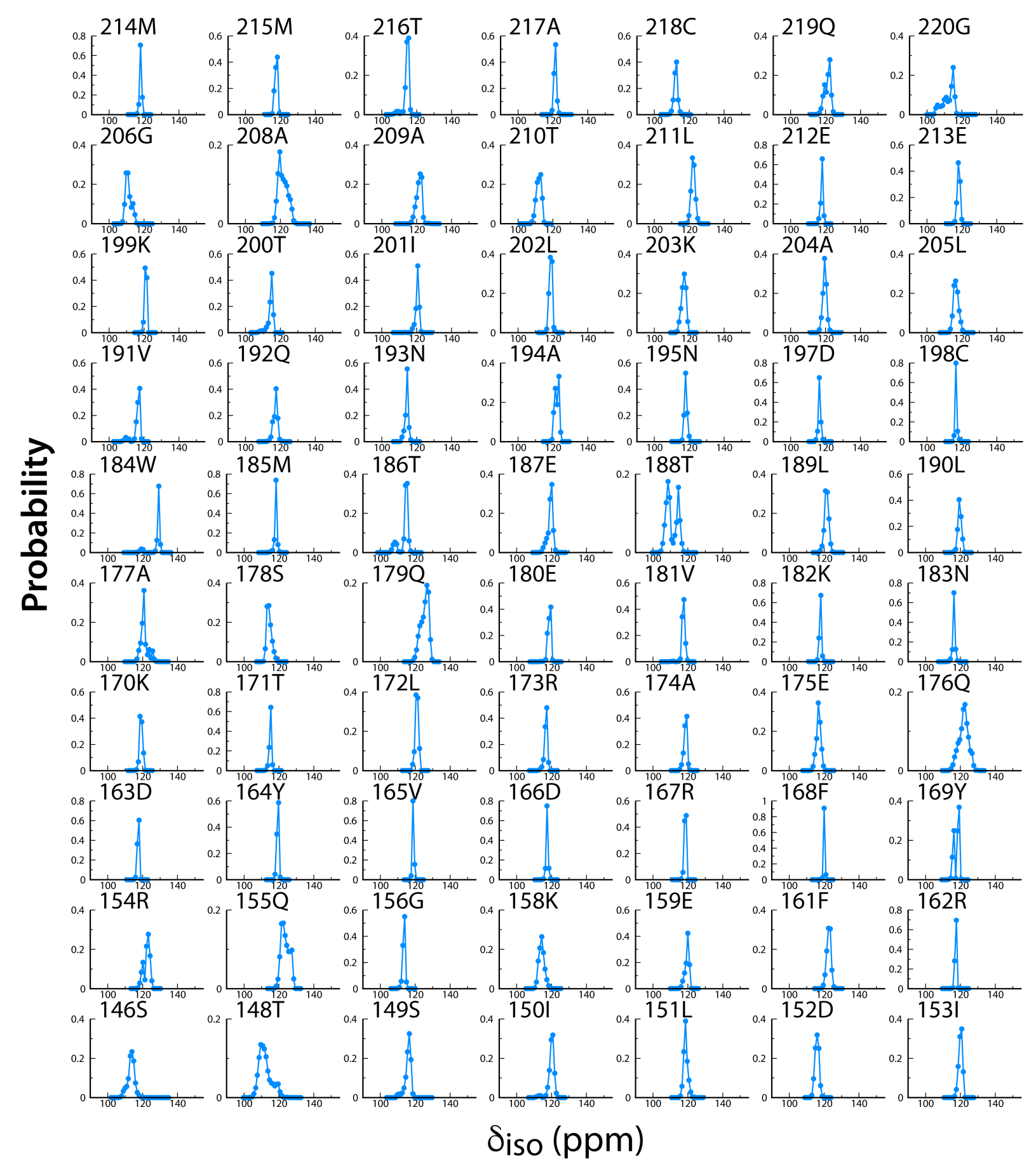

Figure S6. (con'd) Probability distributions of ${ }^{15} \mathrm{~N}$ isotropic chemical shifts of HIV-1 CA calculated by Shiftx based on 5000 frames extracted from 100 ns MD simulation. 
G89
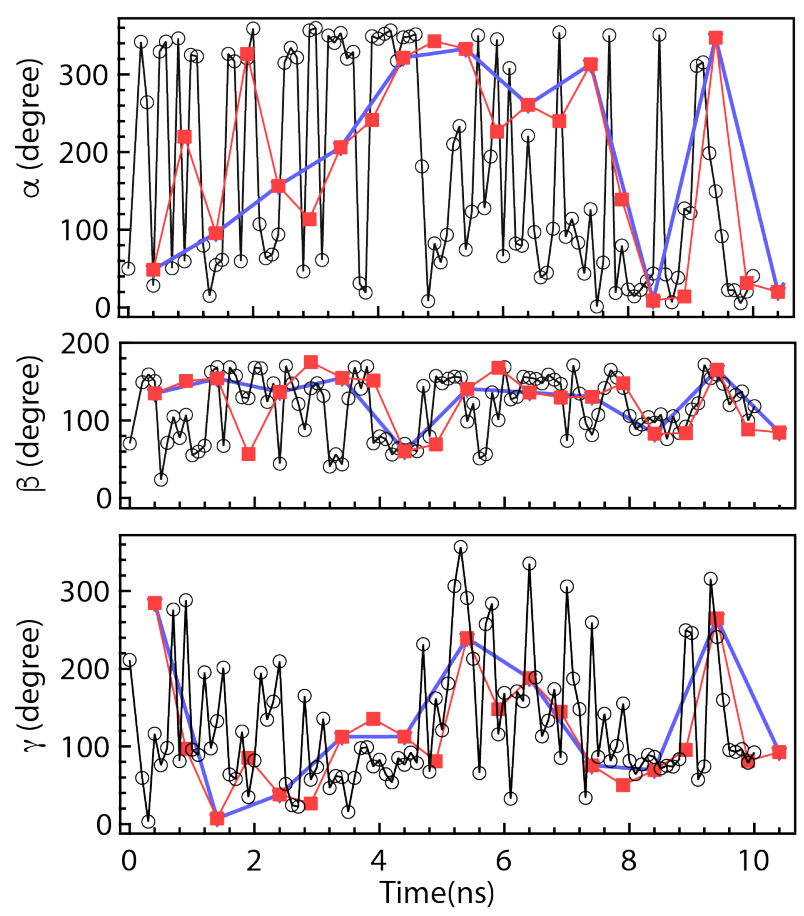

E98

$\rightarrow-100$ frames from 100ns

-100 frames from 10 ns

-200 frames from 100ns
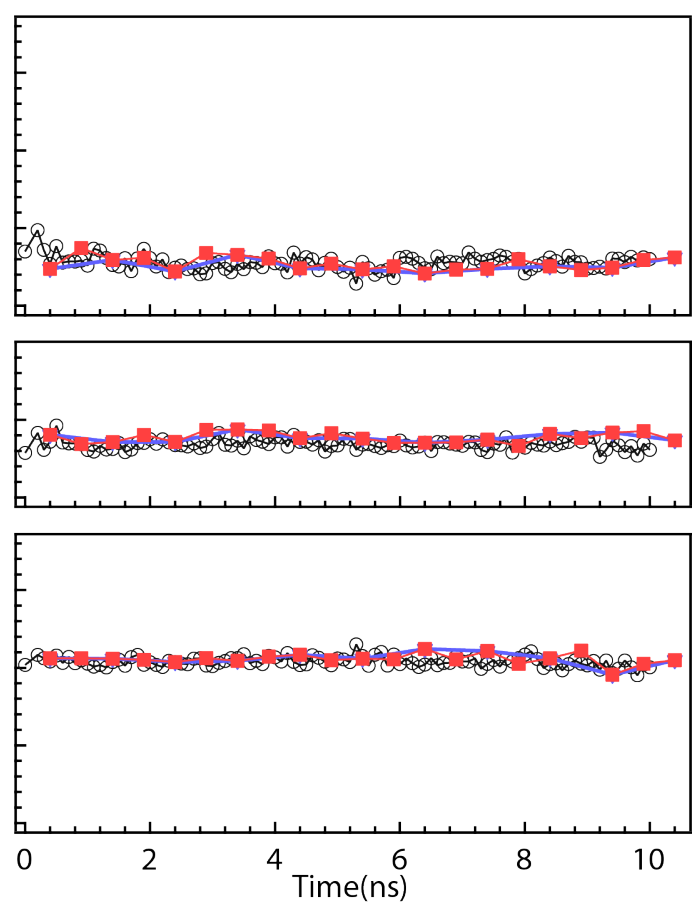

Figure S7. Euler angles of the ${ }^{15} \mathrm{~N}$ CSA tensors for $\mathrm{G} 89$ and $\mathrm{E} 98$ residues of CA, calculated by MD/DFT with different sampling schedules: 200 frames from 100-ns MD trajectory, red; 100 frames from 100-ns MD trajectory, blue; 100 frames from the first 10 ns of the 100-ns MD trajectory, black. The corresponding ${ }^{15} \mathrm{~N}$ CSA parameters for G89 are: $\delta_{\sigma}=28.92 \mathrm{ppm}, \eta_{\sigma}=$ $0.11 ; \delta_{\sigma}=23.28 \mathrm{ppm}, \eta_{\sigma}=0.10 ; \delta_{\sigma}=23.75 \mathrm{ppm}, \eta_{\sigma}=0.12$. The corresponding ${ }^{15} \mathrm{~N}$ CSA parameters for E98 are: $\delta_{\sigma}=93.83 \mathrm{ppm}, \eta_{\sigma}=0.52 ; \delta_{\sigma}=93.00 \mathrm{ppm}, \eta_{\sigma}=0.53 ; \delta_{\sigma}=92.94 \mathrm{ppm}$, $\eta_{\sigma}=0.54$. 

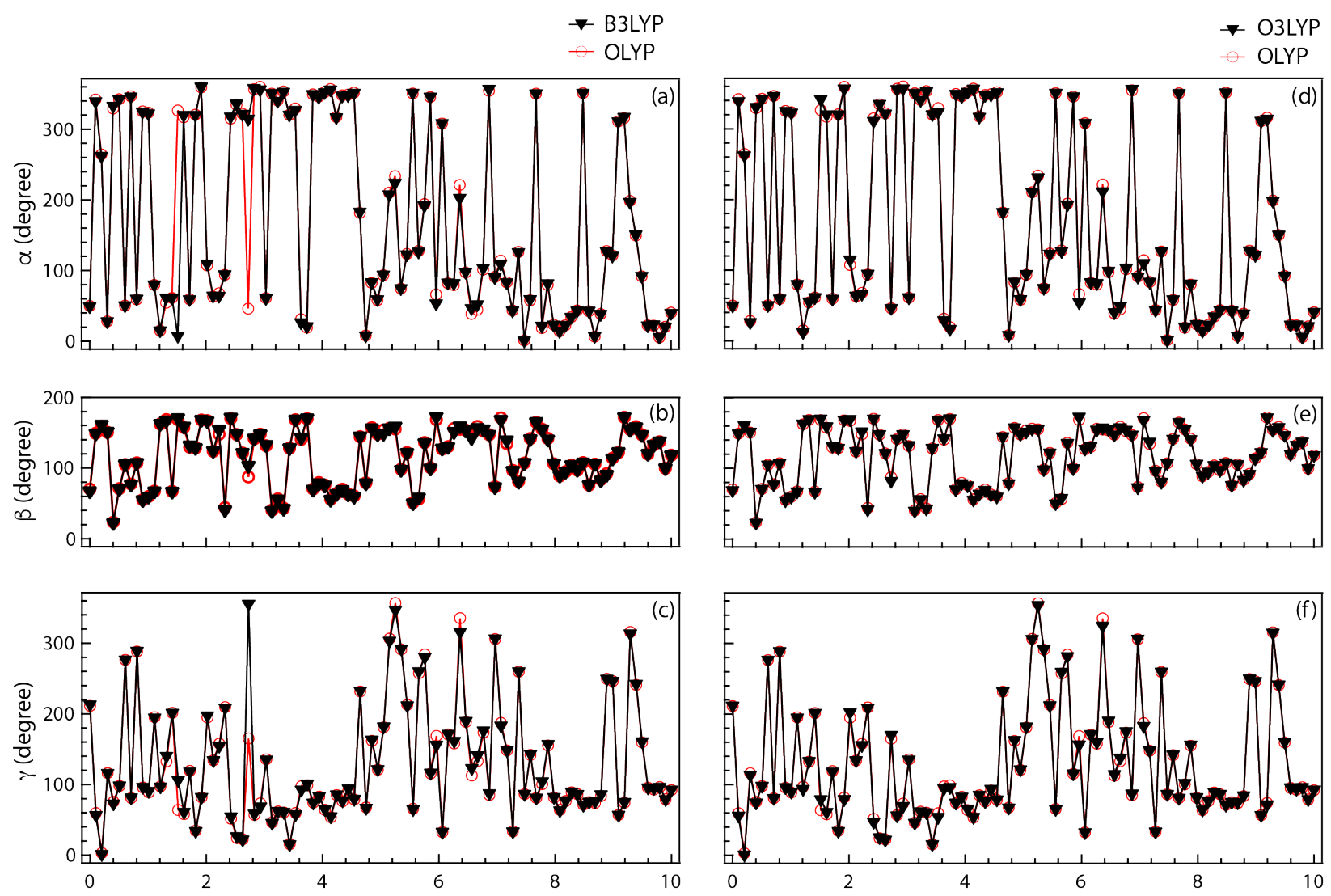

Figure S8. Euler angles of the ${ }^{15} \mathrm{~N}$ CSA tensors for $\mathrm{G} 89$ residue in the molecular frame along the MD trajectory, calculated using (a-c) B3LYP and OLYP functionals (black and red symbols, respectively), and (d-f) O3LYP and OLYP functionals (black and red symbols, respectively). The angles were calculated using $\delta_{\sigma}=25.63,23.75$, and $24.66 \mathrm{ppm}$ for B3LYP, OLYP, and O3LYP, respectively. Note that the differences are small for $\delta_{\sigma}$ computed with the three functionals. For the calculations, 100 frames were used from the first 10 ns of the 100-ns MD trajectory. 